\title{
Modelo Euleriano-Lagrangiano del proceso de gasificación en lecho fluidizado
}

\section{Eulerian-Lagrangian model of the fluidized-bed gasification process}

\author{
DOI: http://doi.org/10.17981/ingecuc.16.1.2020.012
}

Artículo de Investigación Científica. Fecha de Recepción: 27/12/2018. Fecha de Aceptación: 20/11/2019

\author{
Daniel Andrés Quintero Coronel \\ Universidad Francisco de Paula Santander. Ocaña (Colombia) \\ daquinteroc@ufpso.edu.co \\ Franklin Eduardo Consuegra Vargas (id \\ Universidad del Norte. Barranquilla (Colombia) \\ franklinc@uninorte.edu.co \\ Edwin Edgardo Espinel Blanco \\ Universidad Francisco de Paula Santander. Ocaña (Colombia) \\ eeespinelb@ufpso.edu.co
}

Para citar este artículo:

D. Quintero Coronel, F. Consuegra Vargas y E. Espinel Blanco, "Modelo Euleriano-Lagrangiano del proceso de gasificación en lecho fluidizado", INGE CUC, vol. 16, no. 1, pp. 171-179, 2020. DOI: http://doi.org/10.17981/ingecuc.16.1.2020.012

\begin{abstract}
Resumen
Introducción- En procesos de gasificación en lecho fluidizado, es importante evaluar el comportamiento de parámetros de presión, temperatura, composición de gas, características del material a gasificar, flujo de oxidante, entre otros, para llevar a cabo esta evaluación rápidamente, el uso del modelado mediante dinámica de fluidos computacional es una de las herramientas más poderosas. Las fases sólida y gaseosa de la gasificación pueden estimarse mediante el uso un modelo Lagrangiano y Euleriano respectivamente.
\end{abstract}

Objetivo- Desarrollar un modelo mediante el uso de la dinámica de fluidos computacional para la evaluar el comportamiento del proceso de gasificación en lecho fluidizado.

Metodología- A partir de la revisión de la literatura, se seleccionó la geometría del reactor a modelar, las condiciones de operación y el material a gasificar. Se describe detalladamente el modelo desarrollado con el software STAR CCM+, las ecuaciones que rigen el comportamiento del proceso, los estudios de independencia de malla y tiempo, entre otros parámetros de interés.

Resultados- Se validó el modelo desarrollado con datos reportados en la literatura mediante comparación directa de las concentraciones del gas obtenido cuando se utilizó aire como agente oxidante, así mismo, se muestra la concentración del gas obtenido a lo largo del reactor para tiempos de interés.

Conclusiones- A partir de los resultados obtenidos, se logró una buena aproximación con lo reportado en la literatura, lo que permite evidenciar la validez del modelo desarrollado. Los submodelos utilizados en este trabajo difieren de los reportados anteriormente, por lo que se obtiene algunas diferencias en las fracciones molares de las especies obtenidas, no obstante el modelo predice adecuadamente el comportamiento del proceso.

Palabras claves- Gasificación; Lecho fluidizado; CFD; Euleriano; Lagrangiano

\begin{abstract}
Introduction- In fluidized bed gasification processes, it is important to evaluate the behavior of pressure parameters, temperature, gas composition, characteristics of the material to be gasified, oxidant flow, among other parameters. To carry out this evaluation quickly, the use of computational fluid dynamics modeling is one of the most powerful tools. The solid and gaseous phases of gasification can be estimated using a Lagrangian and Eulerian model respectively.

Objective- To develop a model using computational fluid dynamics to evaluate the behavior of the gasification process in a fluidized bed.

Methodology- From the literature review, the geometry of the reactor to be modeled, the operating conditions and the material to be gasified were selected. The model developed with the STAR CCM+ software, the equations that govern the behavior of the process, the mesh and time independence studies, among other parameters of interest, are described in detail.

Results- The model developed was validated with data reported in the literature by direct comparison of the concentrations of the gas obtained when air was used as an oxidizing agent, as well as showing the concentration of the gas obtained throughout the reactor for times of interest.

Conclusions- From the results obtained, a good approximation was achieved with what is reported in the literature, which allows evidencing the validity of the model developed. The sub-models used in this work differ from those reported previously, so some differences are obtained in the molar fractions of the species obtained, however the model adequately predicts the behavior of the process.
\end{abstract}

Keywords- Gasification; Fluidized bed; CFD; Eulerian; Lagrangian 


\section{INTRODUCCIÓN}

El crecimiento acelerado de la población mundial y asociado a ello la necesidad de satisfacer energéticamente a cada individuo ha provocado que se consuman excesivamente los recursos naturales que el planeta posee. Es claro que la transformación de energía tiene bases fundamentadas en la utilización de combustibles fósiles como principal fuente energética, asociado a esto, las consecuencias ambientales son irreversibles, los combustibles fósiles son los principales causantes de emisiones de gases de efecto invernadero [2]. Por otro lado, el agotamiento de los combustibles fósiles es también irreversible, por tal razón la necesidad de buscar fuentes alternas de energía que tengan bajo impacto sobre el ambiente y que puedan ser sostenibles es de vital importancia.

El carbón mineral, es el combustible fósil más importante sobre la tierra pero también es el más sucio (dada su composición química), además no es renovable y puede agotarse en el futuro [3]. Sin embargo, el uso de este recurso mediante gasificación para la obtención de gas de síntesis es una de las estrategias más prometedoras, pues permite disminuir el material contaminante que genera la combustión del carbón mineral.

La gasificación es el proceso termoquímico por el cual un sustrato carbonoso se transforma mediante una oxidación parcial en un gas combustible que contiene, entre otros compuestos, $\mathrm{H}_{2}, \mathrm{CO}, \mathrm{CH}_{4}, \mathrm{CO}_{2}, \mathrm{y} \mathrm{N}_{2}$ (cuando el oxidante es aire), así como diversas impurezas o contaminantes. El gas obtenido permite su uso en las diversas máquinas de combustión actuales, entre las que se destacan motores de combustión interna, turbinas para producción de electricidad, calderas, combustores, entre otros. También puede usarse para la síntesis de productos de mayor valor añadido, como metanol u otros alcoholes, o hidrocarburos con características del diésel, la gasolina o ceras, mediante la síntesis Fischer-Tropsch [4].

Diversos autores, han desarrollado distintos tipos de modelos con el fin de simular el proceso de gasificación de materias primas carbonosas. Lo que se espera con el modelado de la gasificación, es estimar las propiedades características del proceso, así como el comportamiento del mismo ante sus variables de entrada.

Thakare \& Nene [1] desarrollaron un modelo en CFD para la gasificación en lecho Fluidizado, los autores utilizan la forma Euleriana-Lagrangiana para las fases gaseosas y sólidas respectivamente, con elementos discretos para estimar el comportamiento de las partículas de carbón que son introducidas al gasificador de lecho fluidizado. Los resultados del modelo son validados con datos experimentales. Un trabajo similar fue desarrollado por [5], quienes consideraron la transferencia de calor y masa, la densidad de la partícula, el flujo de gas, las reacciones químicas (homogéneas y heterogéneas) del proceso, en búsqueda de las composiciones de gas, perfil de temperatura, y tasa de consumo de carbón, entre otros, los autores validan su modelo con los resultados publicados por [6]. Trabajos similares han sido desarrollados [7]-[8] y en gasificación en lecho fijo [9].

Mediante el método Euleriano-Euleriano [10] desarrollaron un modelo en el cual dos fases interactúan, la fase primaria (aire) y la fase secundaria (partículas de carbón). El modelo se desarrolló en el software ANSYS/FLUENT. Se simula el comportamiento en dos dimensiones de un gasificador de lecho fluidizado y de flujo arrastrado, para carbón mineral con análisis último y próximo dado. Los autores introducen la etapa de desvolatilización al software, aunque no realizan verificación experimental de sus resultados. Un trabajo similar fue hecho por [11] para la simulación en 2D de la gasificación de biomasa en un planta industrial de Portugal, los autores utilizan el modelo turbulento k-ع. Se desarrollan tres corridas experimentales con el fin de validar los resultados obtenidos con el modelo mediante comparación de las concentraciones de los gases $\mathrm{H}_{2}, \mathrm{CO}_{2}, \mathrm{~N}_{2}, \mathrm{CH}_{4}, \mathrm{CO}$, los autores introducen la etapa de desvolatilización, las reacciones homogéneas y heterogéneas del proceso, así como las condiciones granulares del carbón. Trabajos similares han sido desarrollados por [12].

En este trabajo se desarrolla un modelo de gasificación en lecho fluidizado con el software STAR CCM+, a partir de modificaciones realizadas al modelo planteado por [1], excluyendo el tratamiento de la partícula mediante elementos discretos utilizado por estos, modelando este proceso mediante la fase lagrangiana como partículas multicomponentes de carbón, la cual reduce costo computacional y permite que la simulación se desarrolle con mayor velocidad, sin perder estimaciones representativas del proceso. El trabajo comienza con la descripción del proceso de gasificación en lecho fluidizado, las reacciones de gasificación del proceso, el oxidante usado, así como diversas características generales del mismo. Se hace una descripción de la geometría del reactor, de la materia prima y del agente oxidante, las condiciones iniciales y de frontera del modelo, así como otras características propias de la simulación mediante CFD. Finalmente, se muestran los resultados del modelo y las conclusiones del mismo. Asu vez, este trabajo pretende propiciar las bases para una simulación posterior de un reactor de lecho fijo equicorriente.

\section{A. Descripción del fenómeno}

En los gasificadores de lecho fluidizado, el agente gasificante circula por el interior de los mismos a una velocidad ascensional tal que un lecho de sólido inerte, 
que se añade a su interior, se encuentra en estado de fluidización, existiendo en el interior del gasificador una mezcla que favorece la transferencia de materia y energía entre el combustible y el gas. Por tanto, el sólido pulverizado es mantenido en suspensión mediante el gas. Las partículas minerales se recuperan como cenizas [13]. Estos reactores son adecuados para procesos estacionarios, por tanto, son precisos para instalaciones de pequeña y mediana escala (potencias térmicas entre 10 y 100 MW para reactores de lecho fluidizado atmosféricos y presurizados respectivamente), poseen mayor cantidad de material particulado que los gasificadores de lecho fijo, por lo que se hace necesario que incorporen un ciclón [2].

El proceso de gasificación en general comprende una serie de reacciones químicas homogéneas y heterogéneas, en las cuales el material sólido en presencia de un oxidante produce una combustión incompleta que da lugar a la liberación de energía térmica aprovechada en la etapa de pirólisis para el desprendimiento de volátiles, los cuales a su vez reaccionan con el oxidante y los gases liberados, produciendo el gas final. Las reacciones al interior del lecho son exotérmicas y endotérmicas, desarrolladas en fase sólida y gaseosa, lo cual hace al proceso complejo debido a su propia naturaleza. Este gas está compuesto principalmente por $\mathrm{CO}, \mathrm{CO}_{2}, \mathrm{H}_{2}, \mathrm{CH}_{4}, \mathrm{~N}_{2}$ (el $\mathrm{N}_{2}$ en caso de gasificación usando aire como oxidante) y trazas de otros compuestos [14], [15].

Las reacciones que en este trabajo se tienen en cuenta vienen dadas por:

\section{1) Oxidación de Char}

$$
\begin{aligned}
& 2 \mathrm{char}+\mathrm{O}_{2} \rightarrow 2 \mathrm{CO} \\
& \text { char }+\mathrm{H}_{2} \mathrm{O} \rightarrow \mathrm{CO}+\mathrm{H}_{2} \\
& \text { char }+\mathrm{CO}_{2} \rightarrow 2 \mathrm{CO}
\end{aligned}
$$

\section{2) Reacciones Homogéneas}

$$
\begin{aligned}
& \text { Volátiles }+0.0076 \mathrm{O}_{2} \rightarrow 0.417 \mathrm{CO}_{2}+0.583 \mathrm{CH}_{2}+0.021 \mathrm{~N}_{2} \text { (4) } \\
& \mathrm{CO}+0.5 \mathrm{O}_{2} \rightarrow \mathrm{CO}_{2} \\
& \mathrm{CO}+\mathrm{H}_{2} \mathrm{O} \rightarrow \mathrm{CO}_{2}+\mathrm{H}_{2} \\
& \mathrm{H}_{2}+0.5 \mathrm{O}_{2} \rightarrow \mathrm{H}_{2} \mathrm{O} \\
& \mathrm{CH}_{4}+\mathrm{H}_{2} \mathrm{O} \rightarrow \mathrm{CO}+3 \mathrm{H}_{2}
\end{aligned}
$$

De la reacción (4) volátiles está compuesto por $\mathrm{CH}_{2.332} \mathrm{O}_{0.265} \mathrm{~N}_{0.042}$, para el balance estequiométrico.
Dependiendo del agente oxidante, las reacciones químicas varían, pues algunas veces la gasificación puede realizarse con vapor de agua o mezclas de vapor de agua y aire, en el primer caso se espera aumentar el contenido de hidrógeno del gas producto, aunque esto implica suministrar calor desde una fuente externa, con el fin de que puedan llevarse a cabo las reacciones del proceso, por otro lado, cuando se realizan mezclas vapor y aire, se espera mantener en cierto grado la gasificación auto térmica, sin embargo se busca también aumentar el contenido de hidrógeno de los gases.

El modelo que en este trabajo se desarrolla utiliza aire como agente oxidante, éste suministra la cantidad de oxígeno necesaria para que se lleven a cabo las reacciones, el nitrógeno se considera como gas inerte que no reacciona, pero resta energía al proceso.

Las composiciones típicas de los gases producto varían con respecto a la relación de equivalencia, tamaño de partícula, agente oxidante, tipo de material a gasificar entre otras [16]. La Fig. 1 muestra las composiciones típicas de los gases para gasificación con aire en reactores de lecho fluidizado operando a presión atmosférica. Esta composición corresponde a carbón mineral Lignito, el cual posee un porcentaje de carbono alrededor del $60 \%$, volátiles $40 \%$ entre otras características.

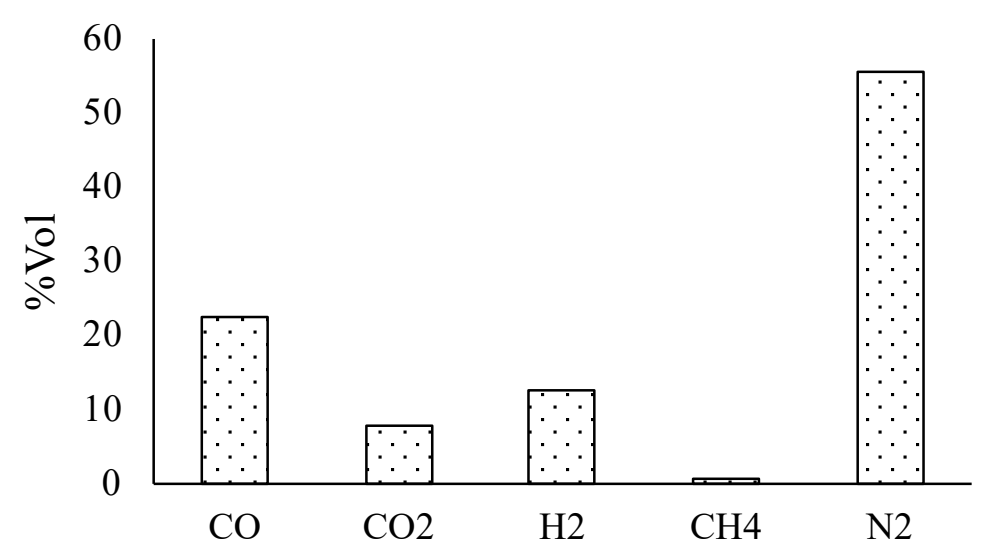

Fig. 1. Composición típica de gas de gasificación. Fuente: [17].

\section{Metodología}

En esta etapa, se describe la geometría del gasificador de lecho fluidizado utilizado, el material a gasificar, la cinética de las reacciones, los modelos de la malla que se seleccionaron, el estudio de independencia de malla, los modelos seleccionados en la simulación y las ecuaciones que gobiernan el modelo.

\section{A. Geometría seleccionada}

La Fig. 2 muestra la geometría del gasificador de lecho fluidizado que se ha modelado, y corresponde a la utilizada por [1], con una ligera modificación realizada a la sección de entrada de material. Las dimensiones del reactor presentan una sección para entrada de aire de $250 \mathrm{~mm}$ diámetro, una para entrada de carbón de $80 \mathrm{~mm}$, una sección de salida de $350 \mathrm{~mm}$ de diámetro y una altura de $4000 \mathrm{~mm}$. 


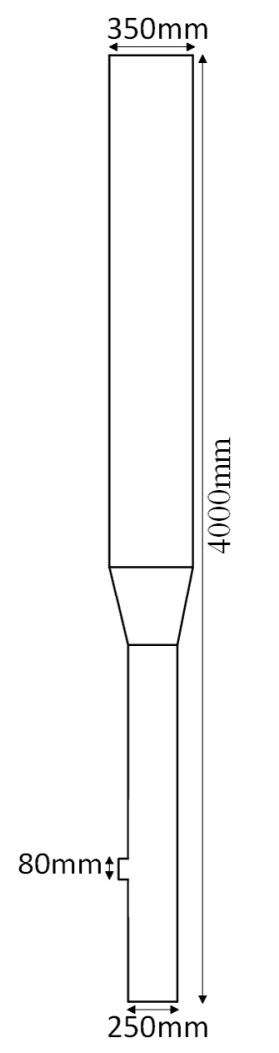

Fig. 2. Geometría seleccionada. Fuente: Autores [1]

\section{B. Carbón utilizado}

Las características del carbón utilizado se muestran en la Tabla 1, y corresponden a las reportadas por [1].

Tabla 1. Características del carbón utilizado.

\begin{tabular}{|l|l|l|l|}
\hline \multicolumn{2}{|c|}{ Análisis último \% } & \multicolumn{2}{|c|}{ Análisis próximo \% } \\
\hline $\mathrm{C}$ & 67.03 & Humedad & 15.23 \\
\hline $\mathrm{H}$ & 5.01 & Volátiles & 40.15 \\
\hline $\mathrm{N}$ & 1.16 & Carbono fijo & 40.66 \\
\hline $\mathrm{S}$ & 1.12 & \multirow{2}{*}{ Cenizas } & 3.96 \\
\hline $\mathrm{O}$ & 21.01 & & \\
\hline
\end{tabular}

Fuente: [1].

\section{Cinética de las reacciones}

Se ha utilizado la ecuación de Arrhenius (9), la cual muestra la dependencia de la cinética de las reacciones químicas con respecto a la temperatura de la reacción en particular.

$$
k(T)=A \cdot e^{\frac{E a}{R T}}
$$

Donde:

$k(T)$ : Constante cinética dependiente de la temperatura.

A: Factor pre-exponencial o factor de frecuencia, indica la frecuencia de las colisiones.

$E a$ : Energía de activación.

$R$ : Constante universal de los gases.

$T$ : Temperatura absoluta.

La Tabla 2 resume los valores para el factor preexponencial y energía de activación.
TABLA 2. ENERGía DE ACTIVACIÓN Y FACTOR PRE-EXPONENCIAL.

\begin{tabular}{|l|l|l|}
\hline \multicolumn{1}{|c|}{ Reacción } & \multicolumn{1}{c|}{$\mathrm{Ea}(\mathrm{J} / \mathrm{kmol})$} & \multicolumn{1}{c|}{$\mathrm{A}$} \\
\hline$(1)$ & $9.29 \mathrm{E} 7$ & 2.3 \\
\hline$(2)$ & $1.47 \mathrm{E} 8$ & 1.33 \\
\hline$(3)$ & $1.3 \mathrm{E} 8$ & 3.149 \\
\hline$(4)$ & $2.027 \mathrm{E} 8$ & $2.119 \mathrm{E} 11$ \\
\hline$(5)$ & $1.7 \mathrm{E} 8$ & $2.239 \mathrm{E} 12$ \\
\hline$(6)$ & $2.88 \mathrm{E} 8$ & $2.25 \mathrm{E} 10$ \\
\hline$(7)$ & $3.1 \mathrm{E} 7$ & $9.87 \mathrm{E} 8$ \\
\hline$(8)$ & $2.09 \mathrm{E} 8$ & $5.922 \mathrm{E} 8$ \\
\hline
\end{tabular}

Fuente: [1].

\section{Mallado de la geometría}

Los modelos y los parámetros seleccionados para el mallado del sistema se especifican en la Tabla 3, la Fig. 3 muestra el mallado de la geometría. El trabajo se desarrolló para una malla bidimensional.

TABla 3. CARACTERÍSTICAS DE LA MALLA SELECCIONADA.

\begin{tabular}{|l|l|}
\hline \multicolumn{2}{|c|}{ Mallado } \\
\hline Model & Surface Remesher \\
\hline Mesh Type & Polyhedral Mesher \\
\hline Base Size & $20 \mathrm{~mm}$ \\
\hline Relative minimum Size & $10 \%$ \\
\hline Relative Target Size & $100 \%$ \\
\hline Prism Layer Thickness & $33.33 \%$ \\
\hline $\mathrm{N}^{\circ}$ Prism Layer & 5 \\
\hline Surface Curvature & 36 \\
\hline Surface Growth Rate & 1.3 \\
\hline
\end{tabular}

Fuente: Autores.

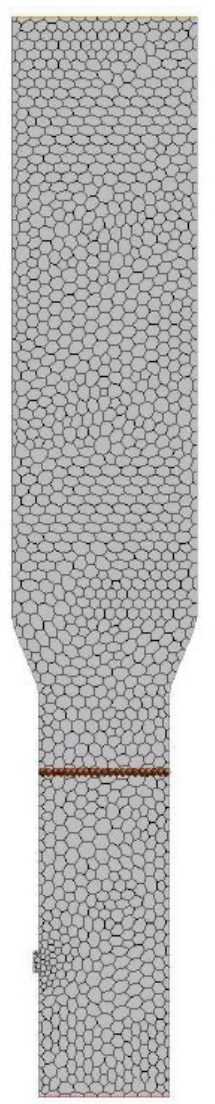

Fig. 3. Mallado de la geometría Fuente: Autores. 


\section{E. Independencia de malla}

El estudio de independencia de malla se llevó acabo para cinco diferentes tamaños de malla, la variable seleccionada para su evaluación corresponde a la fracción molar de $\mathrm{H}_{2}$ sobre una línea de prueba de 20 puntos (Fig. 3). La Tabla 4 y la Fig. 4 muestran el número de celdas correspondientes a cada tamaño de malla y el comportamiento de la fracción molar sobre la línea de prueba respectivamente.

TABLA 4. VARIACIÓN EN LOS PARÁMETROS DE LA MALLA.

\begin{tabular}{|l|l|l|l|l|l|}
\hline \multicolumn{1}{|c|}{ Tamaño base } & $60 \mathrm{~mm}$ & $50 \mathrm{~mm}$ & $40 \mathrm{~mm}$ & $30 \mathrm{~mm}$ & $20 \mathrm{~mm}$ \\
\hline Celdas & 9198 & 11602 & 18006 & 37202 & 113646 \\
\hline Fracción molar & 0.2042 & 0.1851 & 0.1899 & 0.162 & 0.1739 \\
\hline $\begin{array}{l}\text { \% Error (tamaño } \\
\text { base anterior) }\end{array}$ & 10.32 & 2.53 & 17.22 & 6.84 & - \\
\hline
\end{tabular}

Fuente: Autores.

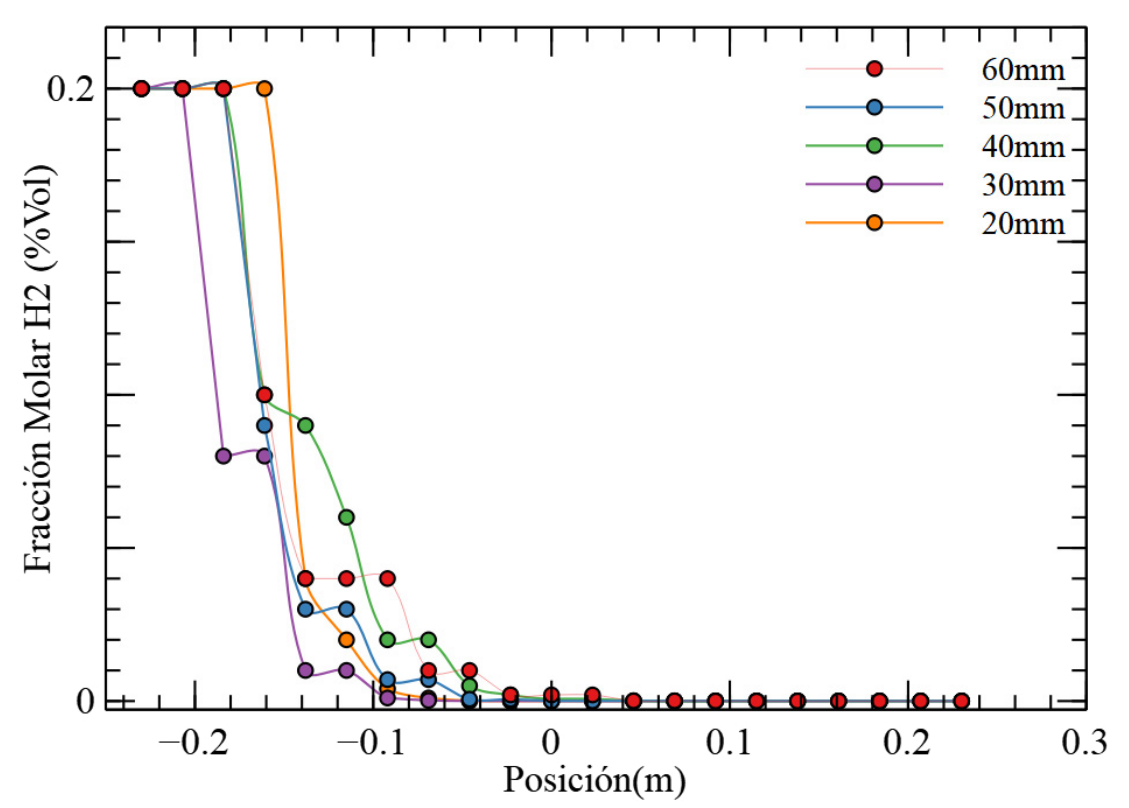

Fig. 4. Comportamiento de la fracción molar $\mathrm{H}_{2}$ sobre la línea de prueba. Fuente: Autores.

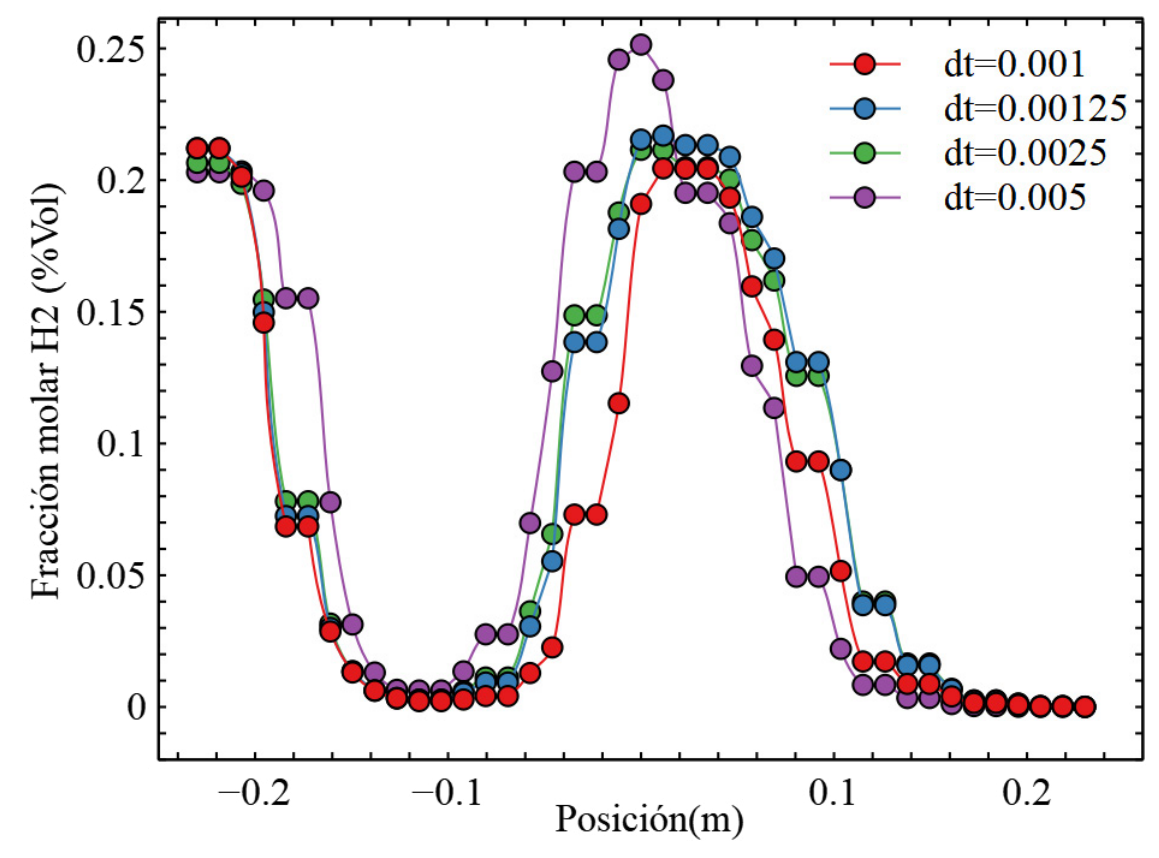

Fig. 5. Comportamiento de la fracción molar $\mathrm{H}_{2}$ sobre la línea de prueba para independencia de tiempo. Fuente: Autores.
Con el objetivo de comparar los resultados reportados por [1], la malla correspondiente a un tamaño base de $20 \mathrm{~mm}$ se seleccionó.

\section{F. Independencia de tiempo}

La prueba de independencia de tiempo se llevó a cabo para los pasos de tiempo 0.001s, $0.00125 \mathrm{~s}, 0.0025 \mathrm{~s} \mathrm{y}$ para 0.005 s. La Fig. 5 muestra el comportamiento de la fracción molar de $\mathrm{H}_{2}$ para una línea de prueba de 20 puntos.

Tomando en consideración los resultados obtenidos en el estudio de independencia de tiempo, se encontró que el paso de tiempo de $0.0025 \mathrm{~s}$ es adecuado para comparar los resultados del modelo desarrollado con los reportados por [1].

\section{G. Ecuaciones que gobiernan el modelo}

El balance de masa a través de un volumen de control es expresado por la ecuación de continuidad.

$$
\frac{\partial \rho}{\partial t}+\nabla \cdot(\rho \boldsymbol{V})=0
$$

Donde $\rho$ corresponde a la densidad, y $V$ es la velocidad del continuo en los ejes coordenados.

Para el caso de la ecuación de conservación del momento, la tasa de cambio de momento lineal en un continuo es igual a la resultante de las fuerzas que actúan sobre éste.

$$
\frac{\partial \rho(\boldsymbol{V})}{\partial t}+\nabla \cdot(\rho \boldsymbol{V} \otimes \boldsymbol{V})=\nabla \cdot \boldsymbol{\sigma}+f_{b}
$$

Donde $f_{b}$ corresponde a las fuerzas externas (gravedad, fuerzas centrifugas) por unidad de volumen que actúan sobre el continuo, $\sigma$ es el tensor de esfuerzos, el cual puede expresarse para un fluido mediante (12).

$$
\frac{\partial \rho(\boldsymbol{V})}{\partial t}+\nabla \cdot(\rho \boldsymbol{V} \otimes \boldsymbol{V})=\nabla \cdot(\rho I)+\nabla \cdot T+f_{b}
$$

Por su parte, (13) corresponde a la de conservación de energía, aquí, $E$ corresponde a la energía total por unidad de masa, $q$ es la fuente de calor y $S_{E}$ es la fuente de energía por unidad de volumen.

$$
\frac{\partial \rho(E)}{\partial t}+\nabla \cdot(\rho E \boldsymbol{V})=f_{b} \cdot \boldsymbol{V}+\nabla \cdot(\boldsymbol{V} \cdot \boldsymbol{\sigma})-\nabla \cdot \boldsymbol{q}+S_{E}
$$

Por otro parte, las ecuaciones que gobiernan la fase dispersa corresponden a (14), (15) y (16).

1) Movimiento del tamaño de partícula

$$
\frac{d v}{d t}=\frac{F_{f}}{m}+g
$$


2) Trayectoria de la partícula

$$
\frac{d x_{p}}{d t}=v
$$

3) Temperatura de la partícula

$$
\frac{d T_{d}}{d t}=\frac{1}{m C_{d}}+\left(\dot{Q}+\dot{m} h_{L}\right)
$$

\section{H. Modelo turbulento}

El modelo k-ع ha sido seleccionado dado que éste es preciso cuando la transferencia turbulenta entre fases asume el rol principal, esto es conveniente cuando se trabaja con el modelo de gasificación en lecho fluidizado [11].

Las ecuaciones de transporte pueden definirse como:

$$
\begin{aligned}
& \frac{\partial}{\partial t}(\rho k)+\frac{\partial}{\partial x_{i}}\left(\rho k u_{i}\right)=\frac{\partial}{\partial x_{j}}\left[\left(\mu+\frac{\mu_{t}}{\sigma_{k}}\right)\right]+G_{k}+G_{b}-\rho \varepsilon-Y_{M}+S_{K} \\
& \frac{\partial}{\partial t}(\rho \varepsilon)+\frac{\partial}{\partial x_{i}}\left(\rho \varepsilon u_{i}\right)=\frac{\partial}{\partial x_{j}}\left[\left(\mu+\frac{\mu_{t}}{\sigma_{\varepsilon}}\right) \frac{\partial \varepsilon}{\partial x_{j}}\right] \\
& +C_{1 \varepsilon} \frac{\varepsilon}{k}\left(G_{k}+C_{3 \varepsilon} G_{b}\right)-C_{2 \varepsilon} \rho \frac{\varepsilon^{2}}{k}+S_{e}
\end{aligned}
$$

Donde:

$C_{1 \varepsilon}, C_{2 \varepsilon}, C_{3 \varepsilon}$ : Constantes

$G_{b}$ : Es la generación de energía cinética turbulenta debido al empuje.

$u$ : Es la velocidad media

$\mu$ : Es la viscosidad

$G_{k}$ : Es la generación de energía cinética turbulenta debido a los gradientes de velocidad media.

\section{Submodelos}

En esta parte de hace una breve descripción de los submodelos utilizados para llevar a cabo este trabajo.

Eddy Break Up (EBU) Model: Este modelo asume que las reacciones están limitadas con la tasa con que la turbulencia puede mezclar los reactivos y calentar la zona de flama. El modelo EBU limita las velocidades de reacción cinética mediante la mezcla turbulenta a gran escala.

Coal combustión: Este modelo permite simular la combustión, la gasificación, y el transporte de carbón, por medio de reacciones prefijadas. Este modelo contiene además una serie de etapas definidas:

Etapa 1 (Coal Moisture Evaporation), corresponde a la evaporación de la humedad presente en el carbón, el carbón pierde humedad por medio de un modelo cuasi estable de evaporación.
Etapa 2 (Raw Coal Devolatilization), corresponde a la etapa de desvolatilización, define cómo las sustancias combustibles se encuentran disponibles para la combustión. El modelo trae una función predefina, aunque puede definirse si se desea otra función. En el caso que aquí se presenta, se trabajó con la función predefinida por el software.

Etapa 3 (Char oxidation), esta etapa define cómo se realiza la oxidación del carbón, el software incorpora tres tipos de oxidante, $\mathrm{O}_{2}, \mathrm{H}_{2} \mathrm{O}$ y $\mathrm{CO}_{2}$. Asu vez esta incorpora dos subetapas, para el caso que aquí se presenta se seleccionó la primera subetapa (First Order Char Oxidation).

\section{J. Condiciones iniciales y de frontera}

La Tabla 5 y Tabla 6 muestran respectivamente las condiciones iniciales y de frontera bajo las cuales este modelo se desarrolló.

TABla 5. Condiciones INICIALES ESTABLECIDAS.

\begin{tabular}{|l|l|l|}
\hline \multicolumn{1}{|c|}{ Presión } & \multicolumn{2}{c|}{$1 \mathrm{~atm}$} \\
\hline Fracción molar especies & $0.21 \mathrm{O}_{2}$ & $0.79 \mathrm{~N}_{2}$ \\
\hline Velocidad & $0.277 \mathrm{~m} / \mathrm{s}$ \\
\hline
\end{tabular}

Fuente: Autores [1].

TABla 6. Condiciones de Frontera establecidas.

\begin{tabular}{|l|l|l|}
\hline \multicolumn{3}{|c|}{ Entrada de aire } \\
\hline Tipo de frontera & \multicolumn{2}{|l|}{ Entrada de velocidad } \\
\hline Velocidad & $0.277 \mathrm{~m} / \mathrm{s}$ \\
\hline Fracción molar especies & $0.21 \mathrm{O}_{2}$ & $0.79 \mathrm{~N}_{2}$ \\
\hline Temperatura estática & $850^{\circ} \mathrm{C}$ \\
\hline \multirow{2}{|c|}{ Inyector } \\
\hline Tipo de inyector & De superficie \\
\hline Flujo másico & $0.0111 \mathrm{~kg} / \mathrm{s}$ \\
\hline Diámetro partícula & $10 \mu$ \\
\hline & Raw Coal & 0.8081 \\
\cline { 2 - 3 } & Cenizas & 0.0396 \\
\cline { 2 - 3 } & Humedad & 0.1523 \\
\hline Fracción molar especies & $0.001804 \mathrm{~m} / \mathrm{s}$ \\
\hline Velocidad & 1 atm \\
\hline Presión & $0.21 \mathrm{O}_{2}$ & $0.79 \mathrm{~N}_{2}$ \\
\hline Fracción molar especies & $25^{\circ} \mathrm{C}$ \\
\hline Temperatura estática & \multicolumn{2}{|l}{0} \\
\hline
\end{tabular}

Fuente: Autores [1]. 


\section{Resultados}

En al caso de la formación de especies, el monóxido de carbono se produce de las reacciones de oxidación de Char (1, 2, 3), y de las reacciones homogéneas (4) y (8). La Fig. 6 muestra el comportamiento de la fracción molar de $\mathrm{CO}$ a lo largo del gasificador. $\mathrm{El}_{2}$ se produce de las reacciones (2), (6) y (8), mientras el CH4 en la reacción (4).

La Fig. 6 muestra la generación progresiva del CO, el cual es producto de las reacciones heterogéneas representadas en (1) a (3), y las reacciones homogéneas indicadas en (4) y (8) respectivamente. De los gases combustibles, éste es el que se produce en mayor cantidad, con fracciones molares características de procesos de gasificación con aire.

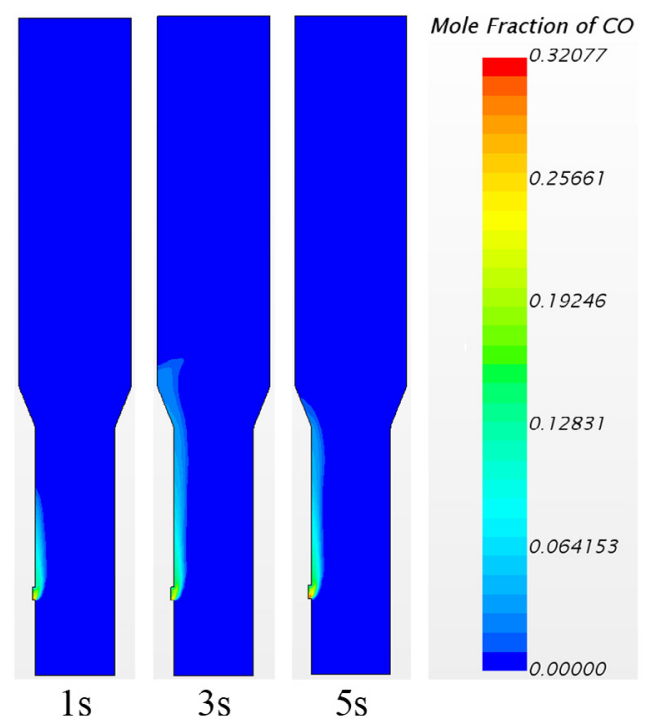

Fig. 6. Comportamiento de la fracción molar CO a lo largo del tiempo. Fuente: Autores.

Por otra parte, la Fig. 7 indica la formación de Hidrógeno, el cual en las primeras etapas del proceso presenta composiciones altas de este gas, formado principalmente por la reacción heterogénea indicada en (2), y por las reacciones homogéneas (6) y (8). No obstante, el hidrógeno disminuye a medida que avanza el proceso, debido a que reacciona para formar agua como lo muestra la Fig. 9.

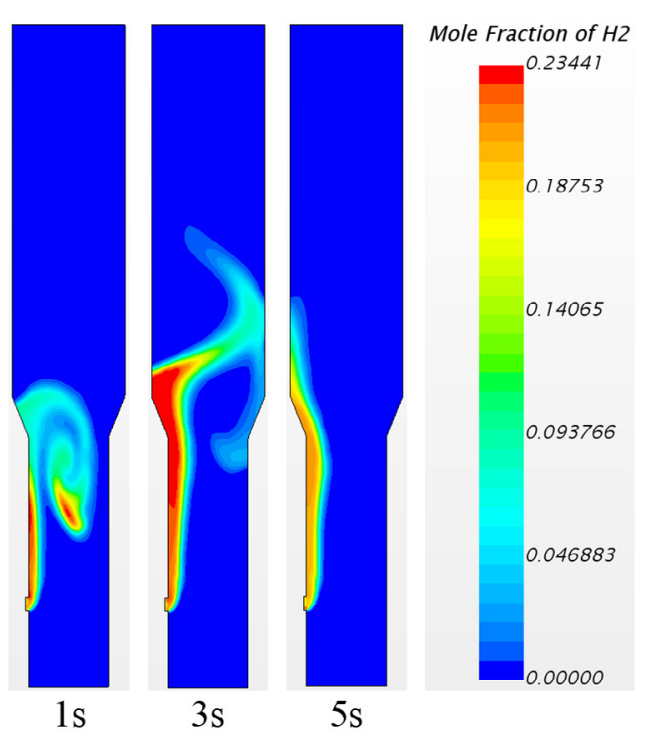

Fig. 7. Comportamiento de la fracción molar $\mathrm{H}_{2}$ a lo largo del tiempo. Fuente: Autores.
Comportamientos similares muestran las Fig. 8, Fig. 10 y Fig. 11, las cuales indican la evolución del $\mathrm{CO}_{2}, \mathrm{O} 2$ y N2 respectivamente. Por su parte, la Fig. 12 muestra la composición del CH4, el cual presenta composiciones bajas, características del proceso de gasificación con aire como oxidante. Esta tendencia es influenciada también debido a las características de la materia prima utilizada (Tabla 1) en el desarrollo del modelo.

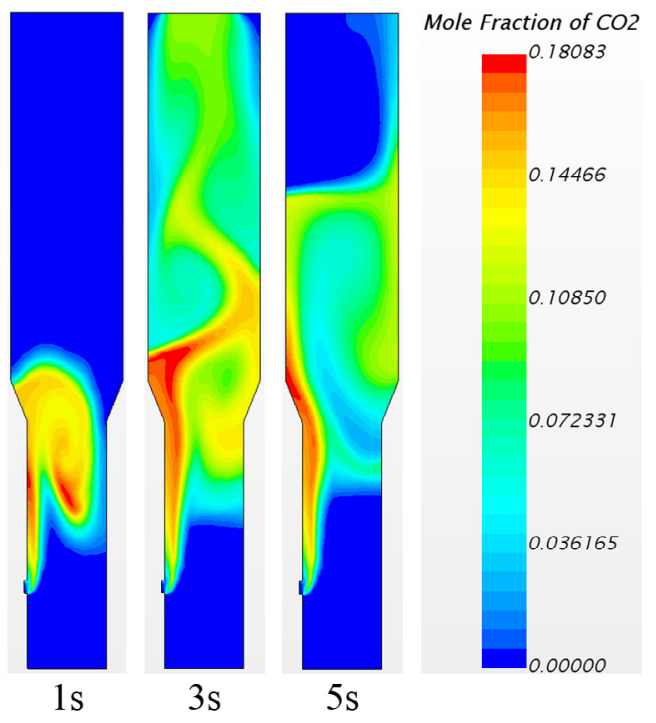

Fig. 8. Comportamiento de la fracción molar $\mathrm{CO}_{2}$ a lo largo del tiempo. Fuente: Autores.

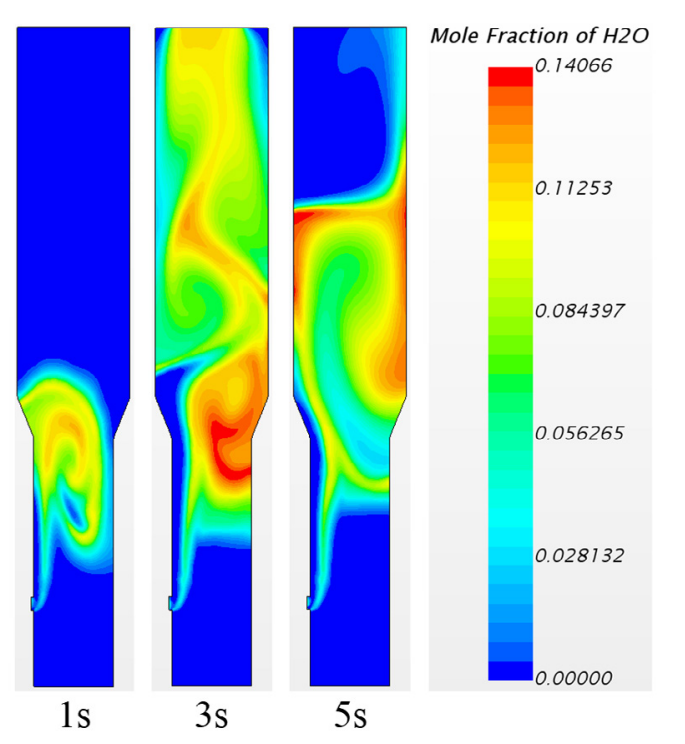

Fig. 9. Comportamiento de la fracción molar $\mathrm{H}_{2} \mathrm{O}$ a lo largo del tiempo. Fuente: Autores.

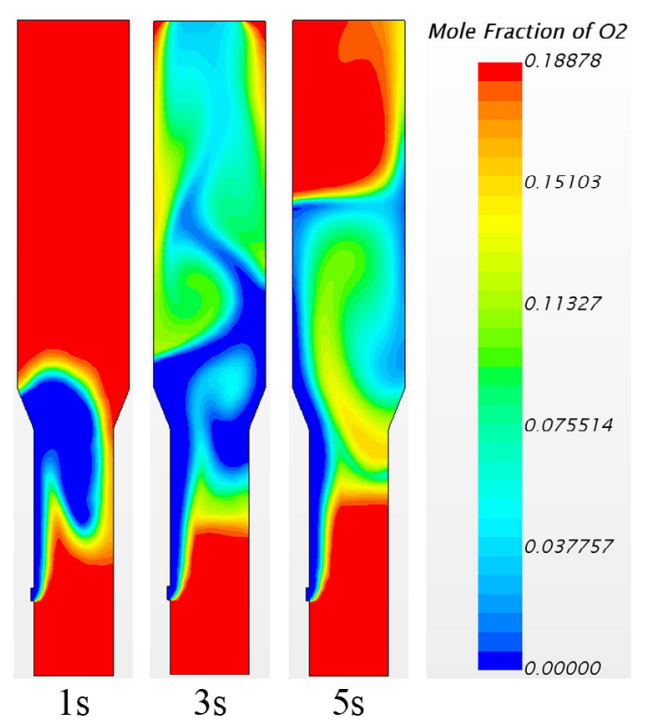

Fig. 10. Comportamiento de la fracción molar $\mathrm{O}_{2}$ a lo largo del tiempo. 


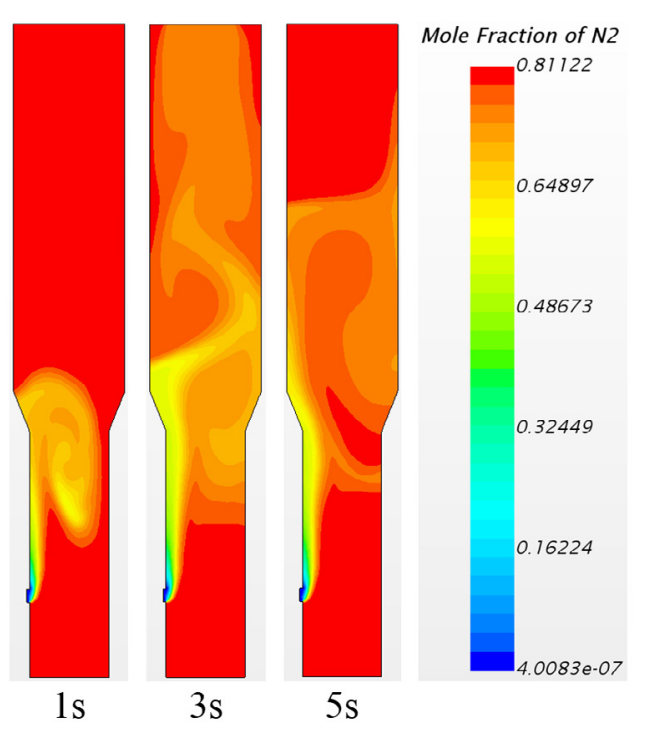

Fig. 11. Comportamiento de la fracción molar $\mathrm{N}_{2}$ a lo largo del tiempo. Fuente: Autores.
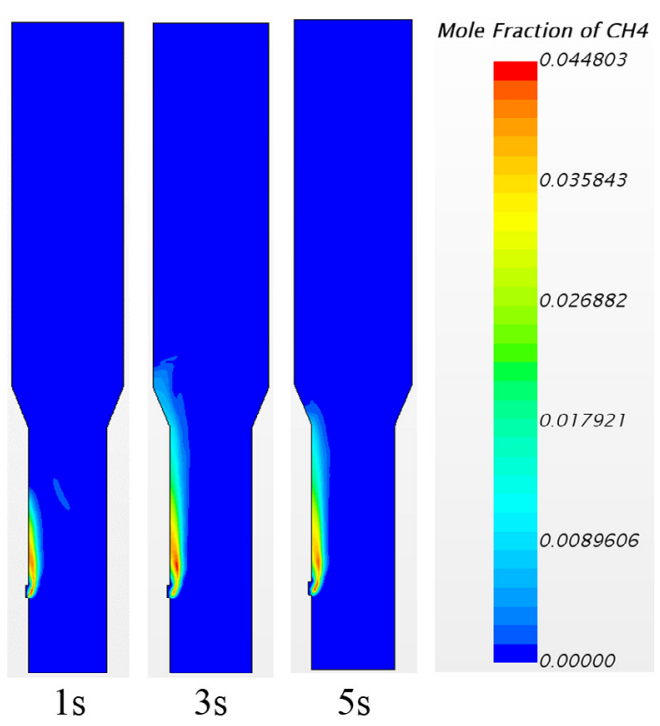

Fig. 12. Comportamiento de la fracción molar $\mathrm{CH}_{4}$ a lo largo del tiempo. Fuente: Autores.

\section{A. Validación}

Se comparó la concentración molar máxima de las especies reportadas por [1] y las encontradas con este trabajo. La Fig. 13 muestra los datos obtenidos, se presenta una ligera sobre estimación de los resultados obtenidos con este modelo, sin embargo, es una buena primera aproximación a trabajos futuros en el proceso de gasificación en lecho fluidizado.

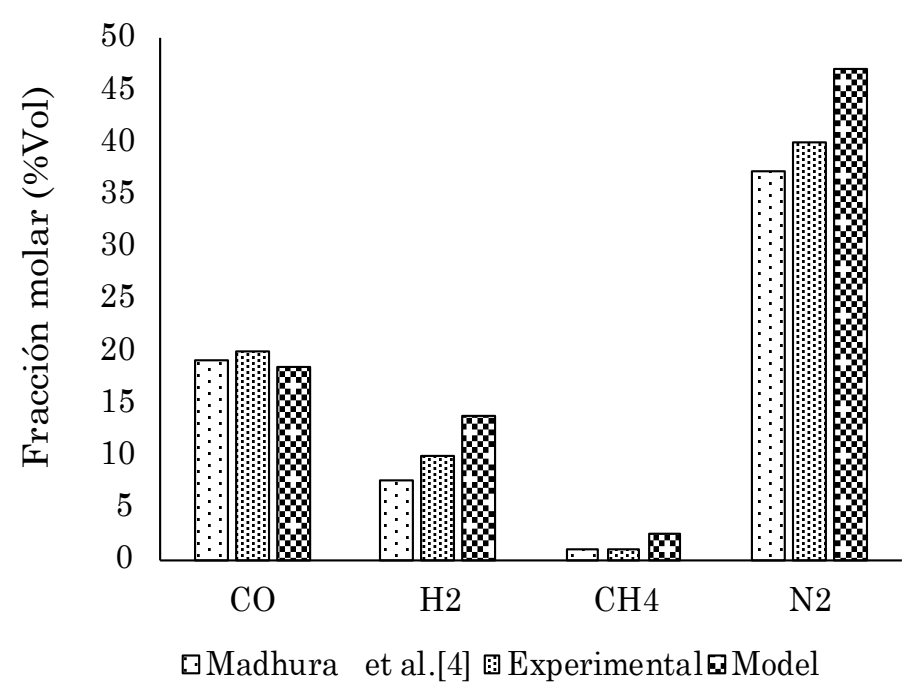

Fig. 13. Comparación resultados del modelo. Fuente: Autores.
Esta sobre estimación de la composición obtenida se debe a los modelos y submodelos utilizados en la simulación por [1] y los empleados en este trabajo. Los resultados encontrados indican composiciones más cercanas cuando se les compara con los valores experimentales utilizados por [1] para la validación de su modelo. Con base en esto, el modelo desarrollado en este trabajo muestra valores representativos de la gasificación en lecho fluidizado, lo que a su vez permitirá su utilización para trabajos futuros en este tipo de reactores.

\section{Conclusiones}

Mediante el modelo desarrollado se lograron obtener composiciones promedio de CO del $18.445 \%$, por su parte el $\mathrm{H}_{2}$ correspondió a $13.64 \%$, en menor medida $\mathrm{CH}_{4}$ con $2.47 \%$ y $\mathrm{N}_{2}$ con $46.90 \%$. Esto permitió obtener un gas con un poder calorífico inferior de $4.7 \mathrm{MJ} /$ $\mathrm{m}^{3}$, parámetro representativo de la gasificación con aire como agente oxidante.

El modelo describe detalladamente los parámetros utilizados para el desarrollo del mismo mediante el uso del Software STAR CCM+, lo cual permite que pueda ser utilizado como una guía en trabajos futuros por otros autores.

El modelado en CFD del proceso de gasificación en lecho fluidizado es una herramienta poderosa en el estudio de los parámetros que afectan al sistema, permite la visualización de variables importantes del sistema, así como el análisis del mismo, lo cual es importante pues logra ajustar y visualizar parámetros que de forma experimental son difíciles de observar y de ajustar.

Los resultados obtenidos con este modelo son una buena aproximación a los que podrían obtenerse en trabajos experimentales, además, permiten analizar el comportamiento de las diversas especies a lo largo del reactor, mediante el uso de especies químicas y reacciones definidas e incorporadas en la librería del software STAR CCM+.

El modelo hace una pequeña modificación a la entrada de carbón lecho al compararlo con el de [1], pues este en su trabajo no reporta la forma del mismo y si está sobre la parte externa o interna del lecho, esto puede afectar la distribución de las especies a lo largo del reactor.

Los valores obtenidos de las fracciones molares difieren en cierta medida a los obtenidos por [1], esto puede deberse a los modelos y sub modelos que se han utilizado en el desarrollo de este trabajo y a los que pudo utilizar [1] , sin embargo este modelo proporciona resultados aproximados que serán la base para trabajos futuros. 


\section{REFERENCIAS}

[1] M. Thakare \& A.A.Nene, "Eulerian-Lagrangian CFD modeling of coal gasification," IERJ, Sp. Ed., pp. 1-9, Jun. 2017.

[2] M. La Villetta, M. Costa \& N. Massarotti, "Modelling approaches to biomass gasification: A review with emphasis on the stoichiometric method," Renew. Sustain. Energy Rev., vol. 74, pp. 71-88, Jul. 2017. https://doi.org/10.1016/j. rser.2017.02.027

[3] A. Franco \& A. R. Diaz, "The future challenges for "clean coal technologies': Joining efficiency increase and pollutant emission control," Energy, vol. 34, no. 3, pp. 348-354, Mar. 2009. https://doi.org/10.1016/j.energy.2008.09.012

[4] G. G. Sáinz, "Estudio experimental de la co- gasificación de fangos de EDAR y carbón con aire en lecho fluidizado," Ph. D Thesis, UNIZAR, Zaragoza, ES, 2013.

[5] J. Xie, W. Zhong, B. Jin, Y. Shao \& Y. Huang, "EulerianLagrangian method for three-dimensional simulation of fluidized bed coal gasification," Adv. Powder Technol., vol. 24, no. 1, pp. 382-392, Jan. 2013. https://doi.org/10.1016/j. apt.2012.09.001

[6] A. Ocampo, E. Arenas, F. Chejne, J. Espinel, C. Londoño, J. Aguirre, J. D. Perez, "An experimental study on gasification of Colombian coal in fluidised bed," Fuel, vol. 82, no. 2, pp. 161-164, Jan. 2003. https://doi.org/10.1016/ S0016-2361(02)00253-3

[7] R. K. Thapa, C. Pfeifer \& B. M. Halvorsen, "Modelling of reaction kinetics in bubbling fluidized bed biomass gasification reactor," Int. J. Energy Environ., vol. 5, no. 1, pp. 35-44, Jan. 2014. https://doi.org/10.2495/HT140161

[8] A. Klimanek, W. Adamczyk, A. Katelbach-Woniak, G. Wecel \& A. Szlek, "Towards a hybrid Eulerian-Lagrangian CFD modeling of coal gasification in a circulating fluidized bed reactor," Fuel, vol. 152, pp. 131-137, Jul. 2015. https://doi.org/10.1016/j.fuel.2014.10.058

[9] R. Jayathilake \& S. Rudra, "Numerical and Experimental Investigation of Equivalence Ratio (ER) and Feedstock Particle Size on Birchwood Gasification," Energies, vol. 10, no. 8, pp. 1-19, Aug. 2017. https://doi.org/10.3390/ en10081232

[10] J. Khan \& T. Wang, "Development of a Devolatilization Model in Multi-Phase Simulation for a Hybrid EntrainedFlow and Fluidized Bed Mild Gasifier," in Proc. 28th Annu. Intl. Pittsburgh Coal Conf, Pittsburgh, PA, Sep. 12-15, 2011, Paper 32-3.

[11] N. Couto, V. Silva, E. Monteiro, P. Brito \& A. Rouboa, "Using an Eulerian-granular 2-D multiphase CFD model to simulate oxygen air enriched gasification of agroindustrial residues," Renew. Energy, vol. 77, no. 1, pp. 174-181, May. 2015. https://doi.org/10.1016/j.renene.2014.11.089

[12] Q. Zhang, L. Dor, W. Yang \& W. Blasiak, "Mathematical modeling of municipal solid waste plasma gasification in a fixed-bed melting reactor," Energ. Fuel, vol. 25, no. 9, pp. 4129-4137, Nov. 2011. https://doi.org/10.1021/ef200383j
[13] I. Sánchez, A. Gallardo \& N. Edo, "Análisis comparativo de las tecnologías de valorización de residuos basadas en la gasificación," in CONAMA 2014, Fundación Conama, Madrid, ES, November 24-27, 2014.

[14] R. L. Bain \& K. Broer, "Gasification", in Thermochemical Processing of Biomass: Conversion into Fuels, Chemicals and Power. New Jersey, USA: Wiley, 2011. https://doi. org/10.1002/9781119990840.ch3

[15] P. Basu, Biomass Gasification and Pyrolysis Practical Design and Theory. Boston, USA: Academic Press, 2010.

[16] J. Werther, "Potentials of Biomass Co-Combustion in Coal-Fired Boilers," in Proc. 20th Intl. Conf. on Fluidized Bed Combustion. Berlin: Springer, 2009.

[17] R. W. Breault, "Gasification processes old and new: A basic review of the major technologies," Energies, vol. 3, no. 2, pp. 216-240, Feb. 2010. https://doi.org/10.3390/en3020216

Daniel Andres Quintero Coronel es Ingeniero mecánico egresado de la Universidad Francisco de Paula Santander y Magister en Ingeniería mecánica en la Universidad del Norte (Barranquilla). Vinculado al grupo de investigación GITYD (Grupo de Investigación en Tecnología y Desarrollo en Ingeniería 2015-actual). https://orcid.org/0000-0003-2822-5984

Franklin Eduardo Consuegra Vargas es Ingeniero Mecánico graduado de la Universidad del Norte (Barranquilla) y Candidato a Magister en Ingeniería Mecánica en la Universidad del Norte en la ciudad de Barranquilla. https://orcid.org/0000-0002-3706-1304

Edwin Edgardo Espinel Blanco es Ingeniero Mecánico de la Universidad Francisco de Paula Santander (Cúcuta, Colombia) y Magister en ingeniería de la Universidad EAFIT (Medellin, Colombia). En el año 2016 inicia su doctorado en ingeniería en la Universidad Pontifica Bolivariana (Medellín, Colombia). Es docente investigador de la Universidad Francisco de Paula Santander en Ocaña, Colombia, adscrito al grupo de investigación y desarrollo en ingeniería (GITYD) como investigador asociado en la clasificación del departamento administrativo de ciencia, tecnología e innovación de Colombia (COLCIENCIAS), ha publicado diversos artículos en revista indexadas y participado como ponente en diversos eventos académicos a nivel internacional. https://orcid.org/0000-0003-4479-2874 